\title{
Pasado, presente y futuro de la documentación en relaciones públicas: el Institute for Public Relations
}

\author{
Por Jordi Xifra
}

\begin{abstract}
Resumen: El Institute for Public Relations es la única organización en el mundo que ha creado una base de datos para consultar y compartir trabajos y resultados de investigación sobre las relaciones públicas. Con más de cincuenta años de historia y con una función documental desde sus inicios, en 2008 presentó el portal Essential Knowledge Project con el propósito de poner a disposición de académicos, estudiantes y profesionales información sobre las relaciones públicas y la comunicación corporativa y establecer un espacio de diálogo para aportar y compartir informaciones e ideas que enriquezcan este conocimiento. Se presenta la estructura y funcionamiento del Institute for Public Relations y del Essential Knowledge Project destacando su notable función documental para las relaciones públicas en particular y la comunicación empresarial en general.

Palabras clave: Documentación, Investigación, Relaciones públicas, Comunicación empresarial, Comunicación corporativa, Portal Essential Knowledge Project, Institute for Public Relations.

Title: Past, present and future of public relations documentation: the Institute for Public Relations

Abstract: The Institute for Public Relations is the only organization in the world which has created a database to consult and share public relations research on studies and results. With over fifty years of history and a records function since its foundation, in 2008 it created the Essential Knowledge Project site in order to make available to scholars, students and particularly practitioners a body of public relations and corporate communications knowledge and establish a dialog space to contribute and share information and ideas that enrich this knowledge. This article presents the Institute for Public Relations and Essential Knowledge Project structure and how they work, highlighting the significant records function in public relations in particular and organizational communication in general.
\end{abstract}

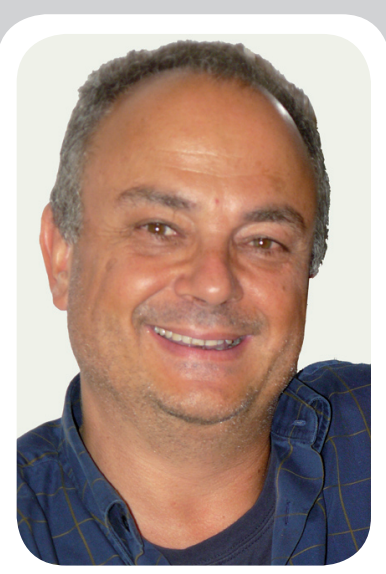

Jordi Xifra es licenciado en derecho por la Universitat de Barcelona y doctor en publicidad y relaciones públicas por la Universitat Autònoma de Barcelona. Profesor titular de Estrategias de Comunicación Corporativa en la Universitat Pompeu Fabra, es miembro del Institute for Public Relations donde ha participado como invitado en sesiones de la Commission on Global Public Relations Research.

Keywords: Documentation, Research, Public relations, Organizational communication, Corporate communications, Essential Knowledge Project portal, Institute for Public Relations.

Xifra, Jordi. "Pasado, presente y fututo de la documentación en relaciones públicas: el Institute for Public Relations". El profesional de la información, 2010, marzo-abril, v. 19, n. 2, pp. 189-193.

DOI: 103145/epi.2010.mar.10

\section{Introducción}

LAS RELACIONES PÚBLICAS y, por extensión, la comunicación empresarial son consideradas por algún autor (Magallón, 2004) como la disciplina invisible de la comunicación.

Esto no sólo es cierto para España, sino también para EUA (Bowen, 2009), donde las relaciones públicas nacieron y evolucionaron, y desde donde se introdujeron en Europa.

Como ocurre con la publicidad (Marcos-Recio; Sánchez-Vigil; Fernández-Fuentes, 2005), los vínculos entre la documentación y las relaciones públicas no son muy estables. El ejercicio de las relaciones públicas es la práctica de la gestión de la comunicación entre las organizaciones y los públicos que conforman el entorno donde evoluciona. La documentación y las relaciones públicas comparten procesos comunicativos donde la información es esencial. Es evidente que la documentación es esencial para la práctica de las relaciones públicas. Las acciones y campañas de relaciones públicas son proyectos estratégicos que se inician con la fase de investigación, con la consiguiente consulta de fuentes documentales diversas, aunque la mayo- ría hacen referencia a publicaciones oficiales, documentos de o sobre la competencia o a información sobre el público o públicos al que se dirigirá el proyecto.

Uno de los aspectos más interesantes -y todavía por explorar- de

"El problema de la investigación en relaciones públicas ha sido siempre la dificultad en el acceso a las principales fuentes documentales" 
la documentación en relaciones públicas (en adelante RPs) se sitúa en el contexto académico y desde una doble perspectiva. En primer lugar, nos referimos a la insuficiencia de documentación académica en comparación con otras disciplinas y, en segundo lugar, al poco uso por parte de los profesionales de esta documentación académica.

El problema de la investigación y la enseñanza de la comunicación empresarial y las RPs en las universidades españolas ha sido siempre la dificultad en el acceso a las fuentes documentales. La creación en 1971 de la licenciatura en Publicidad y Relaciones Públicas -hoy grado en Publicidad y Relaciones Públicas en la mayoría de universidades que ofrecían dicha licenciatura- no se acompañó de una producción científica comparable a otras disciplinas de las ciencias sociales, ni siquiera de las llamadas ciencias de la comunicación (Xifra; Castillo, 2006).

\section{"La documentación y las relaciones públicas comparten procesos comunicativos en los que la información es esencial"}

Esto se ha traducido en el hecho de que tanto investigadores como estudiantes deben utilizar como bases documentales, fuentes extranjeras y sobre todo estadounidenses. Entre ellas destacan la International Academy of Business Disciplines ${ }^{1}$, la Public Relations Society of America ${ }^{2}$, la Public Relations Division de la Association for Education in Journalism \& Mass Communication $(\text { Aejmc })^{3}$ o, en España, Documentación y Relaciones Públicas ${ }^{4}$. En cuanto a las bases de datos, Communication \& mass media complete ${ }^{5}$ y Business source complete $^{6}$ son dos referentes necesarios en este contexto.
La principal fuente documental es la del Institute for Public Relations $^{7}$, que junto con el portal elaborado por el profesor Kirk Hallahan de la Colorado State University de EUA, PR-education.org ${ }^{8}$, constituye la principal fuente documental de investigación y elaboración de un cuerpo teórico y de conocimiento de las RPs en el mundo. Además, el Institute for Public Relations $(I P R)$ es la única organización que tiene entre sus fines solucionar el segundo problema al que hacíamos referencia: el uso documental de la investigación académica por parte de los profesionales del sector.

Este esfuerzo documental ha servido para que los profesionales de la comunicación corporativa (en adelante CC) y las RPs, que hasta finales del siglo XX no sabían dónde encontrar las cada día más numerosas investigaciones sobre las relaciones de las organizaciones con sus públicos, hoy puedan acceder, enriquecer y enriquecerse con ellas (Botan; Hazleton, 2006).

\section{Tradición documental del Institute for Public Relations}

Creado en 1956 con el nombre de Foundation for Public Relations Research and Education -tomando el actual en 1989- es una organización independiente sin ánimo de lucro que sirve de puente entre la academia y la profesión para fomentar la investigación en RPs y la incorporación de este conocimiento a la práctica profesional. De acuerdo con esta misión, su función documental atiende tanto a las necesidades de los académicos como a las de los profesionales. Así lo ha demostrado a lo largo de su más de medio siglo de historia.

En 1959 presentó un programa de becas destinadas a profesores y profesionales. Sin embargo, su vocación documental se inicia en la década siguiente, elaborando en 1964 la primera base de datos de bibliografía y hemerografía de RPs, extendiéndose posteriormente a la documentación en otros soportes diferentes al escrito. En 1968 inició el proyecto de archivo audiovisual para preservar el patrimonio de las RPs y la CC realizando y grabando entrevistas con los principales profesionales. Hoy se conservan 313 entrevistas y conversaciones con los líderes del sector y responsables de departamentos de RPs, CC y/o relaciones institucionales.

\section{"EI IPR es un referente} mundial para

profesionales, académicos

y estudiantes de todo el mundo"

Los empeños audiovisuales no terminaron ahí. En 1973 se hizo cargo de la producción de la película Opinion of the publics, que recogía las principales experiencias y análisis de caso de RPs explicados por sus protagonistas, y que constituye, junto con otro de los documentales producidos por el Institute en 1990, Communications that count ${ }^{9}$, una preciosa fuente documental para la formación práctica.

Además de preocuparse por cuestiones curriculares de los planes de estudios de grado y postgrado, en 1975 lanzó el primer número de la publicación académica Public relations review, que hasta 2008 ha sido la única revista académica del campo de las RPs indexada en el Social science citation index. Desde sus inicios se publican cuatro números anuales y un número especial bibliográfico anual (bibliography issue), donde se recopilan todas las fuentes bibliográficas y hemerográficas relacionadas con las RPs, categorizadas en función de una lista de entradas que van 
desde Activism hasta Writing techniques. Este número especial ha sido durante más de tres décadas la principal base de datos bibliográfica de las RPs.

Con la aparición de internet, el Institute se adaptó al nuevo medio y lo aprovechó para potenciarlo como la primera fuente documental en línea de las RPs mediante su portal.

\section{Portal del IPR}

El principal propósito de la web es impulsar y difundir el cuerpo de conocimiento de las RPs y la CC. Para ello, el portal, que sólo dispone de versión en inglés, presenta la siguiente información:

- Datos sobre el Instituto (organigrama, plan estratégico, financiación, etc.).

- Motor de búsqueda en la base de datos.

- El Essential knowledge project (del que tratamos en el siguiente epígrafe)

- Trabajos de investigación categorizados por temas, en la sección Research (tabla 1).

- Premios

- Eventos

- Noticias de actualidad

Al ser la transmisión de conocimiento la función básica del IPR, la sección Research contiene el mayor archivo de investigaciones de RPs que puede encontrarse en la Red.

Sin embargo, la última y mayor iniciativa documental realizada es la elaboración del Essential Knowledge Project, el portal del que nos ocupamos a continuación y cuyo propósito es doble: por un lado poner a disposición de los profesionales investigaciones académicas y por otro, crear un foro de diálogo para compartir experiencias que ayuden a difundir los resultados de estas aportaciones académicas enriqueciendo a su vez la propia actividad profesional.

\section{Essential knowledge project: portal para generar, consultar y compartir conocimiento}

Se creó en 2008, contribuyendo más que ningún otro empeño del $I P R$ a su definición como fuente de conocimiento fundamental de las RPs. Con esta web se consolida el puente entre la academia y la profesión, se fomenta la investigación y se transmite el conocimiento teórico y práctico. En términos de documentación, el proyecto representa una innovación similar a la publicación en 1964 de la primera bibliografía sobre la investigación en RPs.

Se trata de un portal gratuito (excepto para la descarga de materiales protegidos por derechos de propiedad intelectual) que presenta informes resumen de la investigación realizada sobre determinados temas de interés para profesionales

\begin{tabular}{|l|r|}
\hline \multicolumn{1}{|c|}{ Categorías } & Ítems \\
\hline Publicidad y RPs & 8 \\
\hline Comunicación de crisis & 12 \\
\hline Comunicación intercultural & 5 \\
\hline Comunicación interna & 6 \\
\hline Historia & 4 \\
\hline RPs internacionales & 24 \\
\hline Interdisciplinariedad & 3 \\
\hline Comunicación financiera & 6 \\
\hline Evaluación de campañas y & 51 \\
\hline proyectos & 12 \\
\hline Relaciones con los medios & 20 \\
\hline Nuevas tecnologías y RPs & 1 \\
\hline Indicadores no financieros & 1 \\
\hline Derecho y RPs & 23 \\
\hline RPs y gestión empresarial & 3 \\
\hline Relaciones institucionales & 2 \\
\hline Diccionarios y bibliografías & 15 \\
\hline Gestión de relaciones & 25 \\
\hline Reputación y confianza & 15 \\
\hline Casos de investigación & 24 \\
\hline Métodos de investigación & 23 \\
\hline
\end{tabular}

Tabla 1. Información de la base de datos de investigaciones del Institute for Public Relations y académicos. Cada informe contiene los enlaces a las fuentes originales, de manera que el portal tiene dos niveles de lectura: el general y el específico mediante los enlaces a las investigaciones citadas.

En la página principal se encuentran las entradas a cada temas de investigación:

- Teoría del cambio (y cómo afecta a la CC)

- Agendas consolidadas de investigación

- Responsabilidad social corporativa

- Comunicación de crisis

- Diversidad y práctica de las RPs

- Comunicación interna

- Ética y práctica de las RPs

- RPs globales

- Comunicación financiera, comunicación proactiva (issues management)

- Nuevas tecnologías y RPs

- Modelos de influencia personal

- Reputación, confianza y credibilidad

En 2010 se incorporará un enlace destinado a análisis de casos, siguiendo la idea de que los temas coincidan con la base de datos Research del mismo instituto, de manera que ambas fuentes documentales sean complementarias, aunque algunos temas se incluyan únicamente en el proyecto Essential Knowledge, al menos en un principio, como ocurre con la teoría del cambio.

\section{"Essential knowledge project aporta informes resumen de las investigaciones realizadas"}




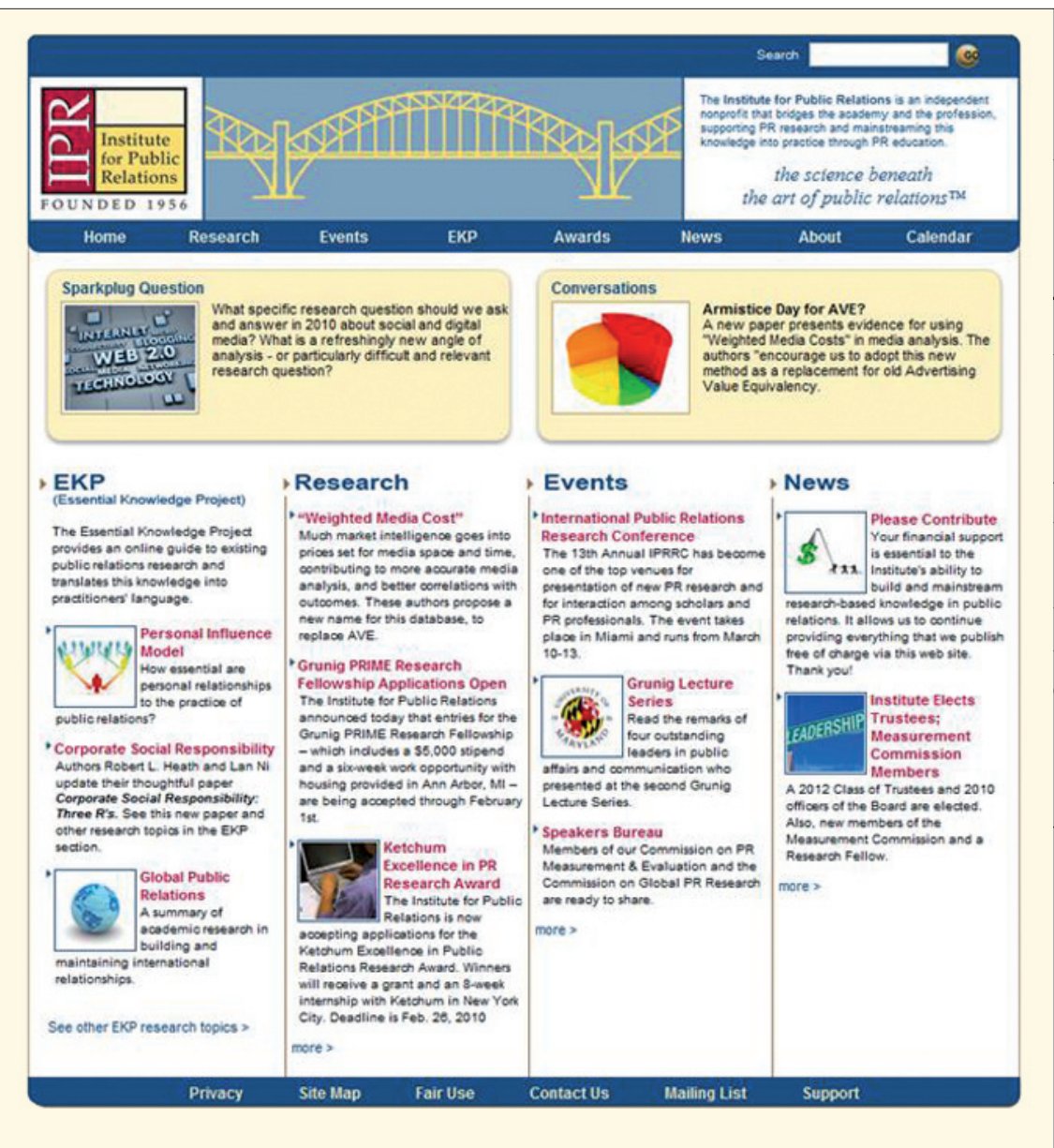

Figura 1. Institute for Public Relations http://www.instituteforpr.org/

Cada informe tiene la estructura de un artículo o capítulo de libro sobre el tema y ha sido elaborado por un académico especialista. Describe cuál es el estado de la investigación, sus antecedentes históricos y sus perspectivas de futuro. Su extensión es de entre 12.000 y 20.000 palabras y toda cita bibliográfica puede consultarse a través del correspondiente enlace.

Los lectores pueden valorar los informes resumen de 1 a 5 , así como aportar comentarios que serán contestados por el autor o autores del informe, generándose un espacio de debate. A partir del diálogo con los visitantes, el académico o académicos encargados del tema incluyen un nuevo texto que actualiza o profundiza en algunos de los aspectos que más han centrado los comentarios. Este enlace se estructura al mismo nivel que el resumen inicial y, a su vez, puede

Todavía es pronto para valorar los resultados de esta iniciativa, pero no cabe duda de que al ofrecer cumentación especializado en RPs y CC. Como base de datos y guía de la investigación académica se ha convertido en el referente para profesionales, académicos y estudiantes de todo el mundo, cubriendo una laguna que bloqueaba seriamente la evolución del conocimiento de esta disciplina.

El conocimiento basado en la investigación es esencial para la maduración de las RPs no sólo como objeto de análisis académico, sino también como profesión encargada de gestionar la CC. Desde esta perspectiva, la función documental del IPR es y será fundamental, mediante proyectos como el Essential knowledge.

\section{Bibliografía}

Botan, Carl; Hazleton, Vincent. "Public relations in a new age". En: Botan, Carl; Hazleton, Vincent. Public relations theory II. Mahwah, New Jersey: Lawrence Erlbaum, 2006, pp. 1-18. ISBN 0-8058-3384-6.

Bowen, Shannon A. "All glamour, no substance? How public relations majors and potential majors in an exemplar program view the industry and function". Public relations review, 2009, Nov., v. 35 , n. 4, pp. 402-410.

Magallón, Sara. "La educación universitaria de las relaciones públicas en España". En: ArceoVacas, José-Luis. Las relaciones públicas en España. Madrid: McGraw-Hill, 2004, pp. 375-388. ISBN 84-481-4183-0.

Marcos-Recio, Juan-Carlos; Sánchez-Vigil, Juan-Miguel; Fernández-Fuentes, Belén.

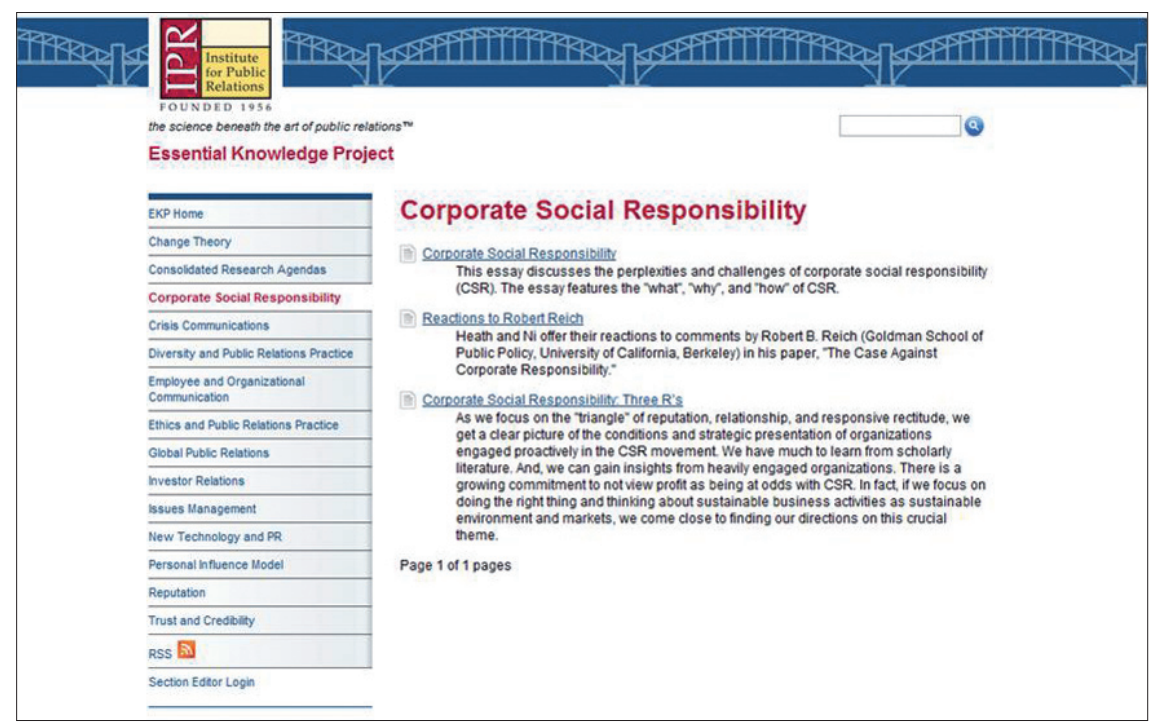

Figura 2. Página del tema "Responsabilidad social corporativa". http://www.instituteforpr.org/essential_knowledge/list/category/Corporate\%20Social\%20Res ponsibility/ 
"Gestión de los contenidos documentales en el ámbito de la publicidad y las relaciones públicas". Scire, 2005, septiembre-diciembre, v. 11, n. 2, pp. 79-98.

Xifra, Jordi; Castillo, Antonio. "Forty years of doctoral public relations research in Spain: A quantitative study of dissertation contribution to theory development". Public relations review, 2006, Sept., v. 32 , n. 3, pp. 302-308
2. http://www.prsa.org/

3. http://aejmc.net/PR/

4. http://multidoc.rediris.es/docrrpp/

5. http://www.ebscohost.com/thisTopic.php?topi $c I D=56 \&$ market $I D=1$

6. http://www.ebscohost.com/thisTopic.php?mar ketID=1\&topicID=399

7. http://www.instituteforpr.org/

8. http://lamar.colostate.edu/ pr/

9. Dirigido principalmente a los altos ejecutivos, este documental recoge ejemplos de cómo las corporaciones utilizan las relaciones públicas en ámbitos como las relaciones institucionales, la gestión comercial y las relaciones de la comunidad.

Jordi Xifra, Departamento de Comunicación, Universidad Pompeu Fabra, Roc Boronat 138, 08018 Barcelona, España. jordi.xifra@upf.edu

\section{0 Análisis de tendencias en información y documentacion}

\section{Algunos de los títulos:}

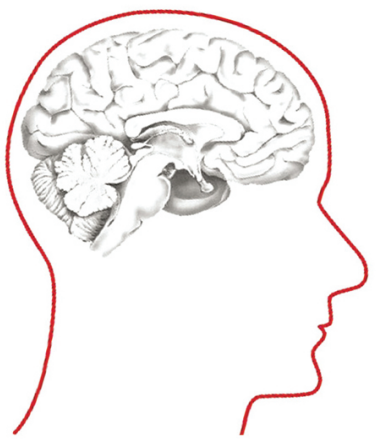

\section{ANUARIO}

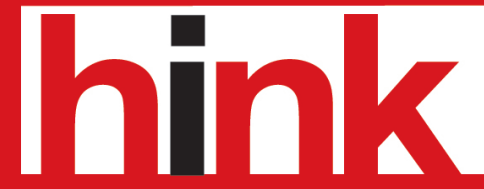

http://www.thinkepi.net/anuario.html 